\title{
COMMITTEES AND SPONSORS
}

\section{Committee Members}

\section{Founders}

Chikayoshi Maeda (Japan)

Wang Liji (China)

\section{International Program Committee}

Chair:

Dae-Im Kang (Korea)

Vice-chairs:

Kazunaga Ueda (Japan)

Zhang Yue (China)

Members:

Cai Changqing (China)

Jin Wan Chung (Korea)

Kitty Fen (Australia)

Toshiyuki Hayashi (Japan)

Lee Shih Mean (Singapore)

John Man (Australia)

Sheau-Shi Pan (Chinese Taipei)

Yon-Kyu Park (Korea)

Chris Sutton (New Zealand)

S.S.K. Titus (India)

Masaaki Ueki (Japan)

Zhang Zhimin (China) 
Committees and Sponsors

\section{National Organizing Committee}

Advisory board:

Jia-Ruey Duann (CMS/ITRI)

Tzeng-Yow Lin (CMS/ITRI)

Chair:

Gwo-Sheng Peng (CMS/ITRI)

Vice-chair:

Wei-En Fu (CMS/ITRI)

Members:

Chiu-Hsien Chen (CMS/ITRI)

Bo-Ching He (CMS/ITRI)

Hsin-Chia Ho (CMS/ITRI)

Yan-Chen Liu (MSL/ITRI)

Hui-Chung Ma (CMS/ITRI)

Sheau-Shi Pan (CMS/ITRI)

Jiunn-Haur Shaw (CMS/ITRI)

Jui-Yiao Su (MSL/ITRI)

Chia-Ling Tsai (CMS/ITRI)

Cheng-Tsair Yang (CMS/ITRI)

Secretary General:

Sheng-Jui Chen (CMS/ITRI)

Executive Secretary:

Chung-Lin Wu (CMS/ITRI) 
List of sponsors:

(in alphabetical order)

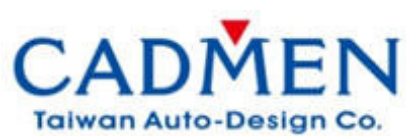

虎門科技股份有限公司
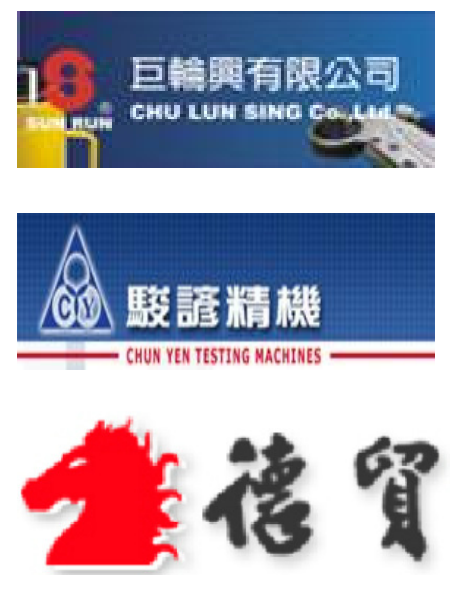

煴昕科技有限公司

\section{Э \\ FORTER TECH. \\ 鼻 颃 科 技}

BIIII Ta

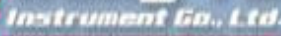

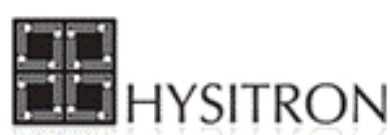

METTLER TOLEDO
CADMEN, Taiwan Auto-Design Co.

Chu Lun Sing Co., Ltd.

Chun Yen Testing Machines

DEAMARK LIMITED

Esense Scientific Ltd.

Forter Tech.

Hung Ta Instrument Co., Ltd.

Hysitron, INC.

Mettler-Toledo 
San Lien Technology Corp. www.sanlien.com

\section{sartoríus sartorius}

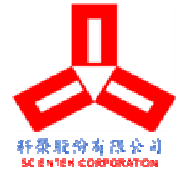

SCIENTEK CORPORATION

(9) SLNHENG

SUNHENG TECHNOLOGY 